\title{
UDOT Automated Freeway Performance Metrics
}

January 26, 2016

Grant Farnsworth, PE

UDOT Traffic Mobility Engineer

Kelly Burns

Eric Rasband

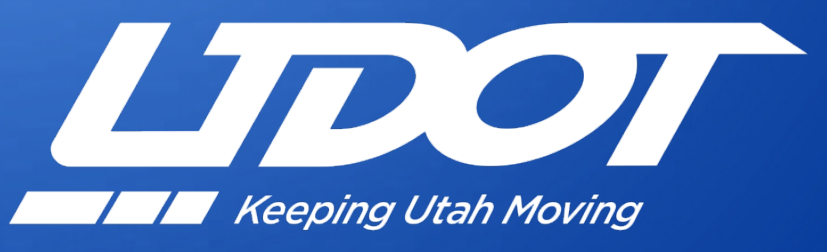




\section{Automated Freeway Performance Measures}

- Project Inception

- Comparison of overall freeway performance

- Objective data vs. anecdotal perception

- Data driven decisions

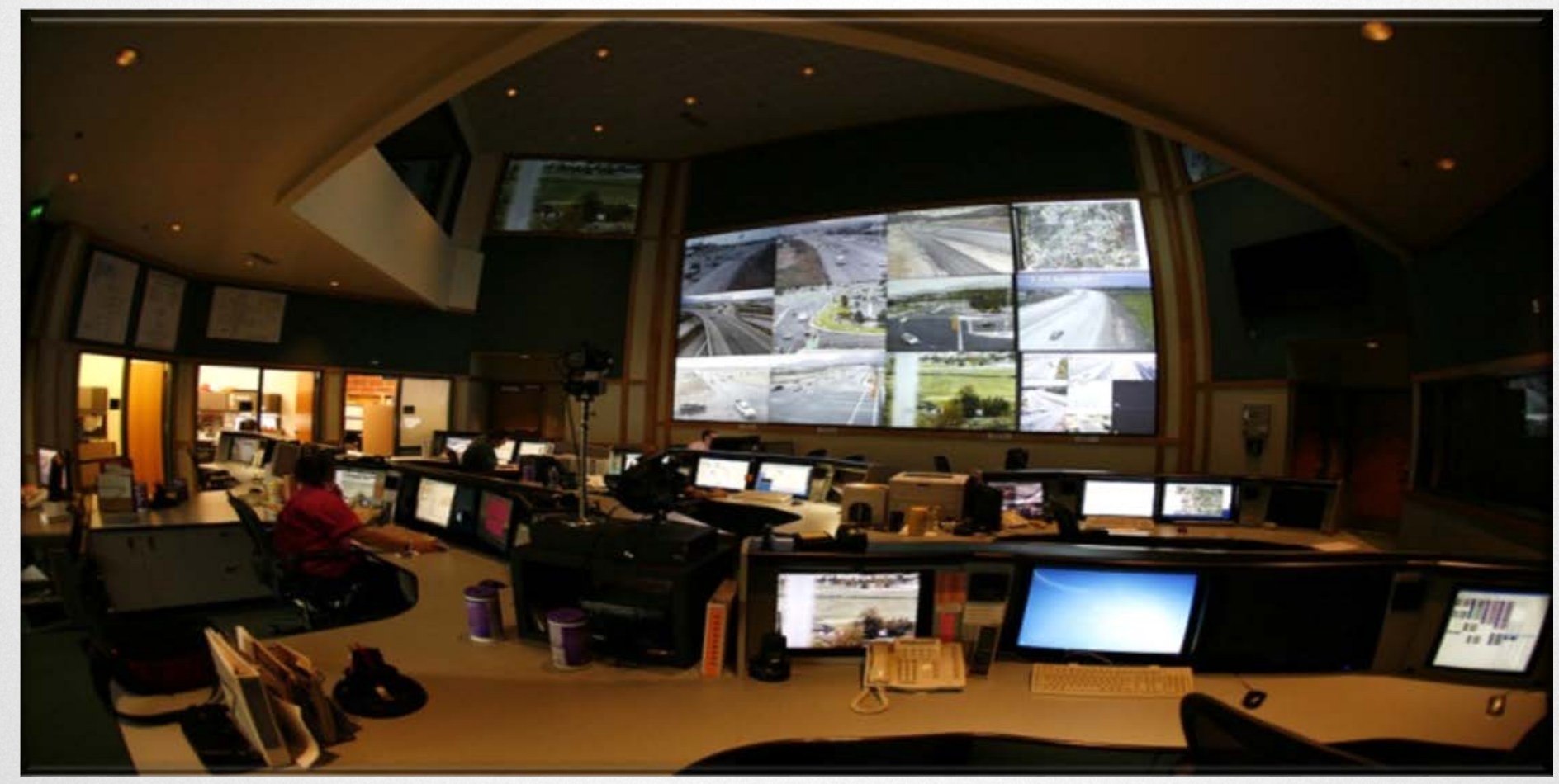




\section{UD0T’s Freeway Detection System}

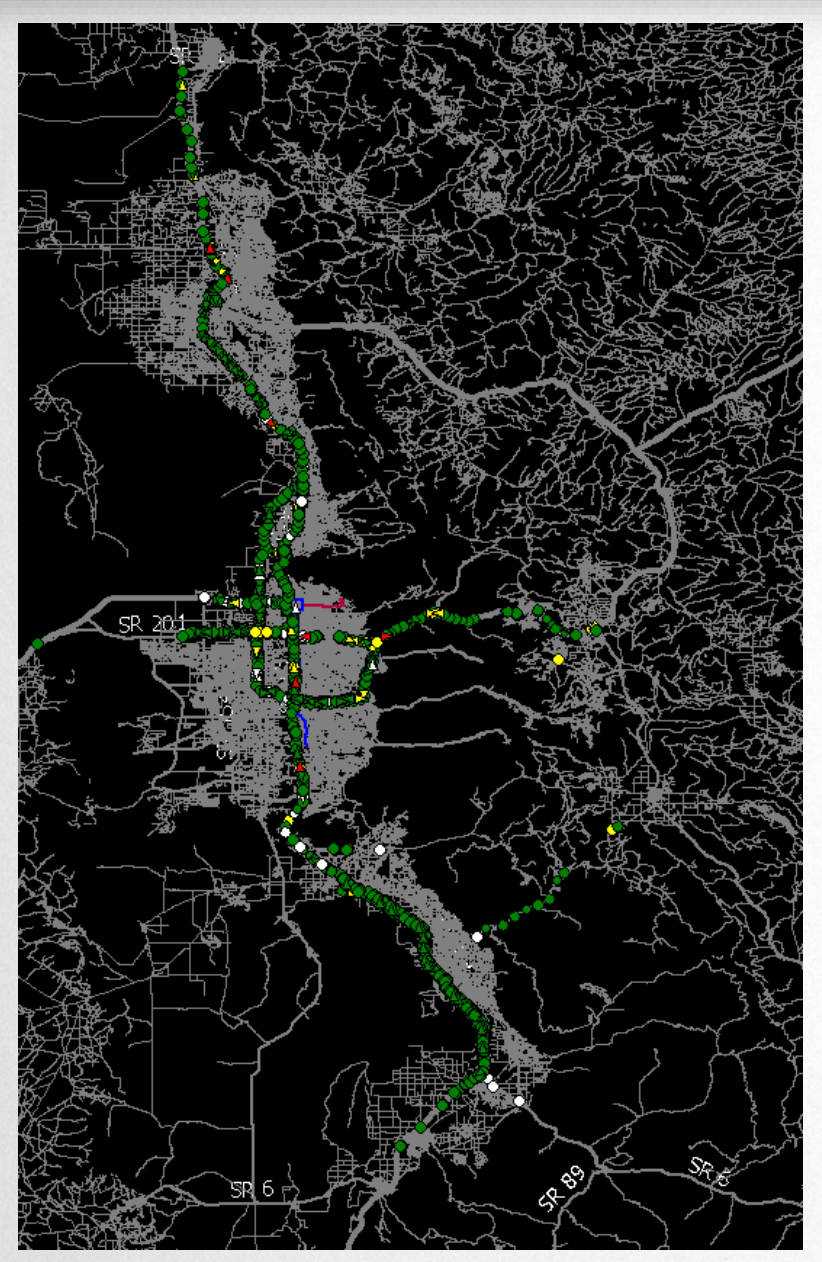

Transuite UDOT Traffic map, travel times, ramp meters

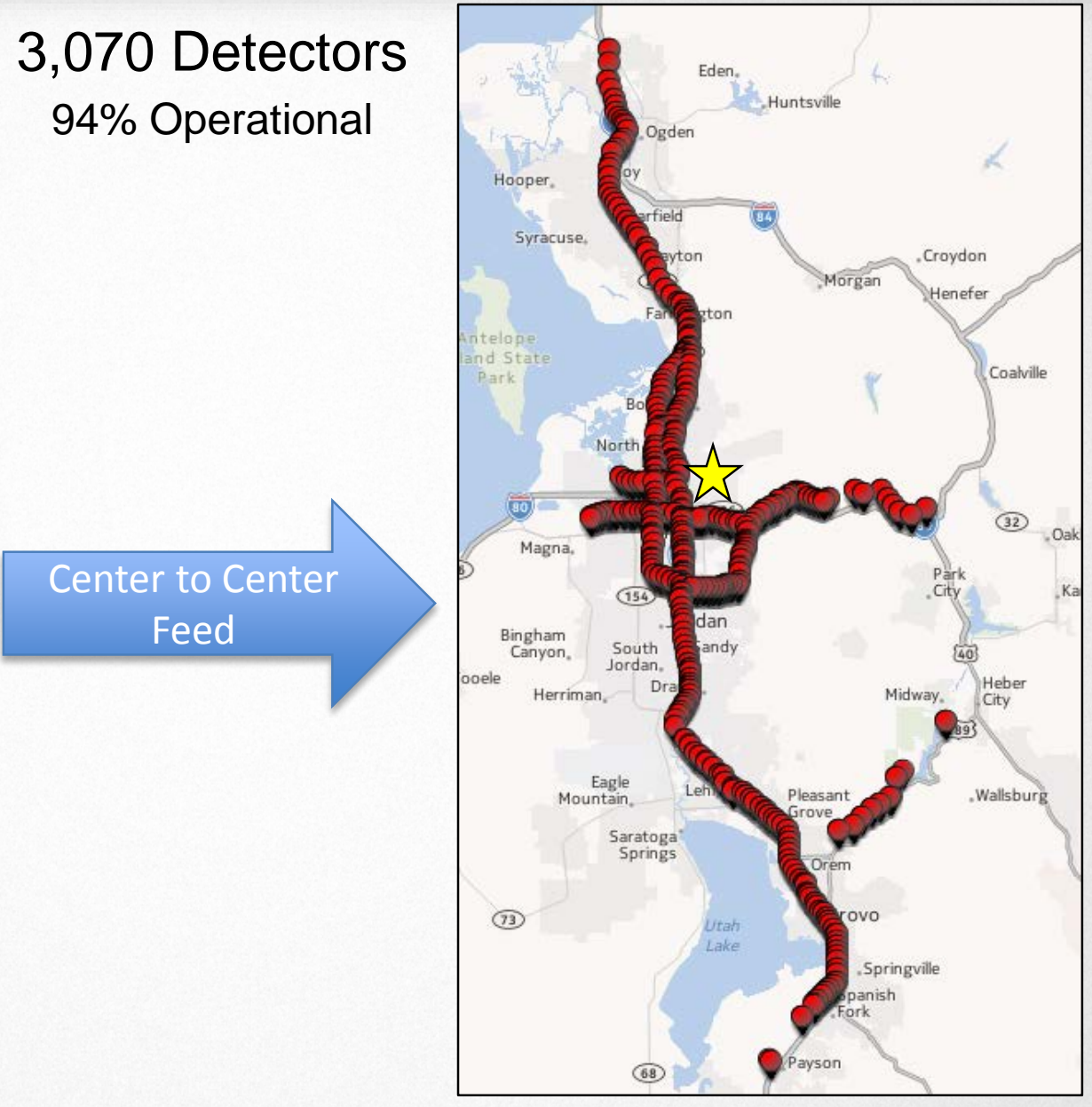

Iteris PeMS (Performance Management System) Data cleaning, historical data aggregation \& analysis 


\section{Umbrella Traffic Performance Metrics Website}

\section{Lper Trafilit Performante Metrites}

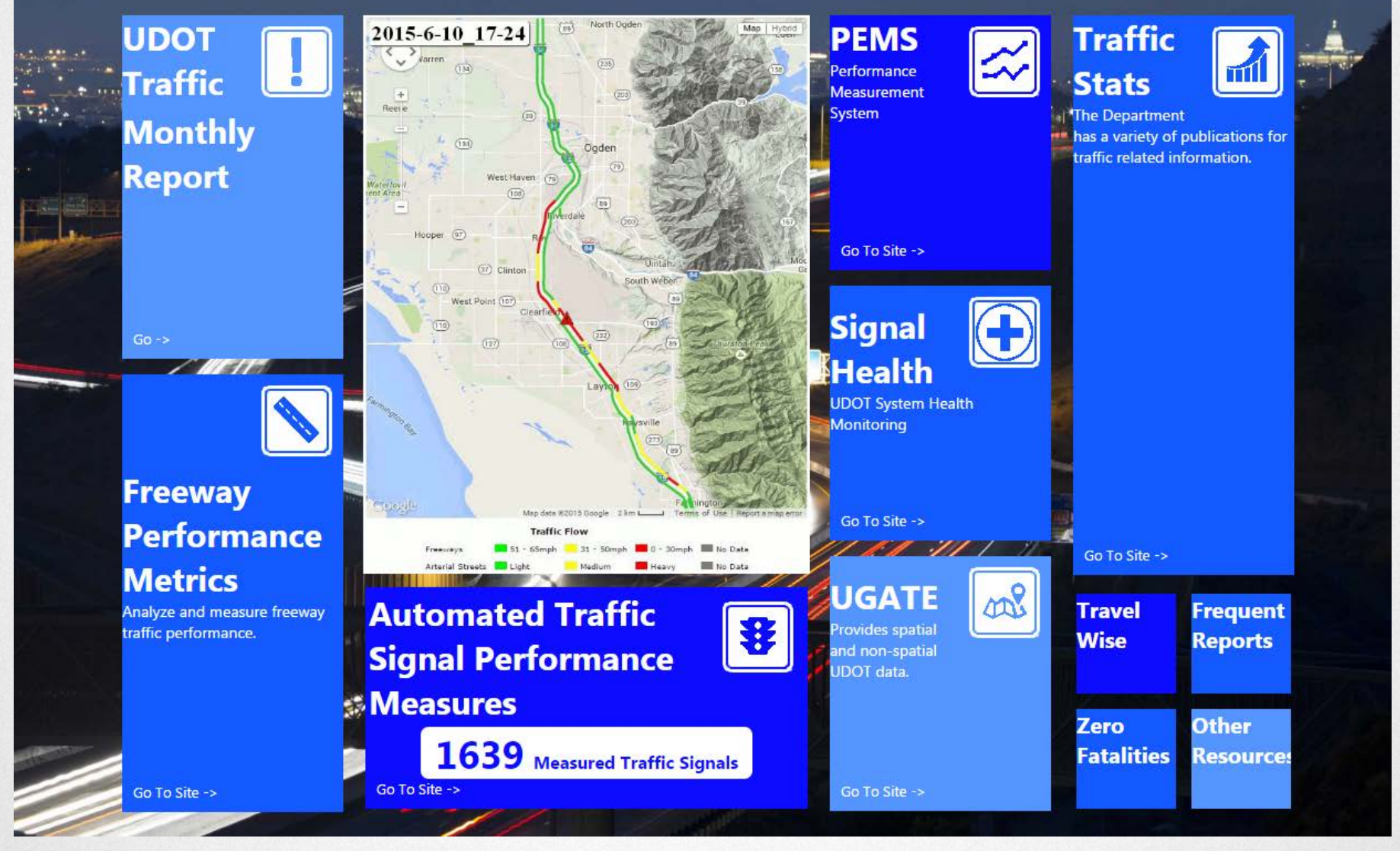

http://udottraffic.utah.gov/performancemetrics/ 
Freeway Performance Metrics Website

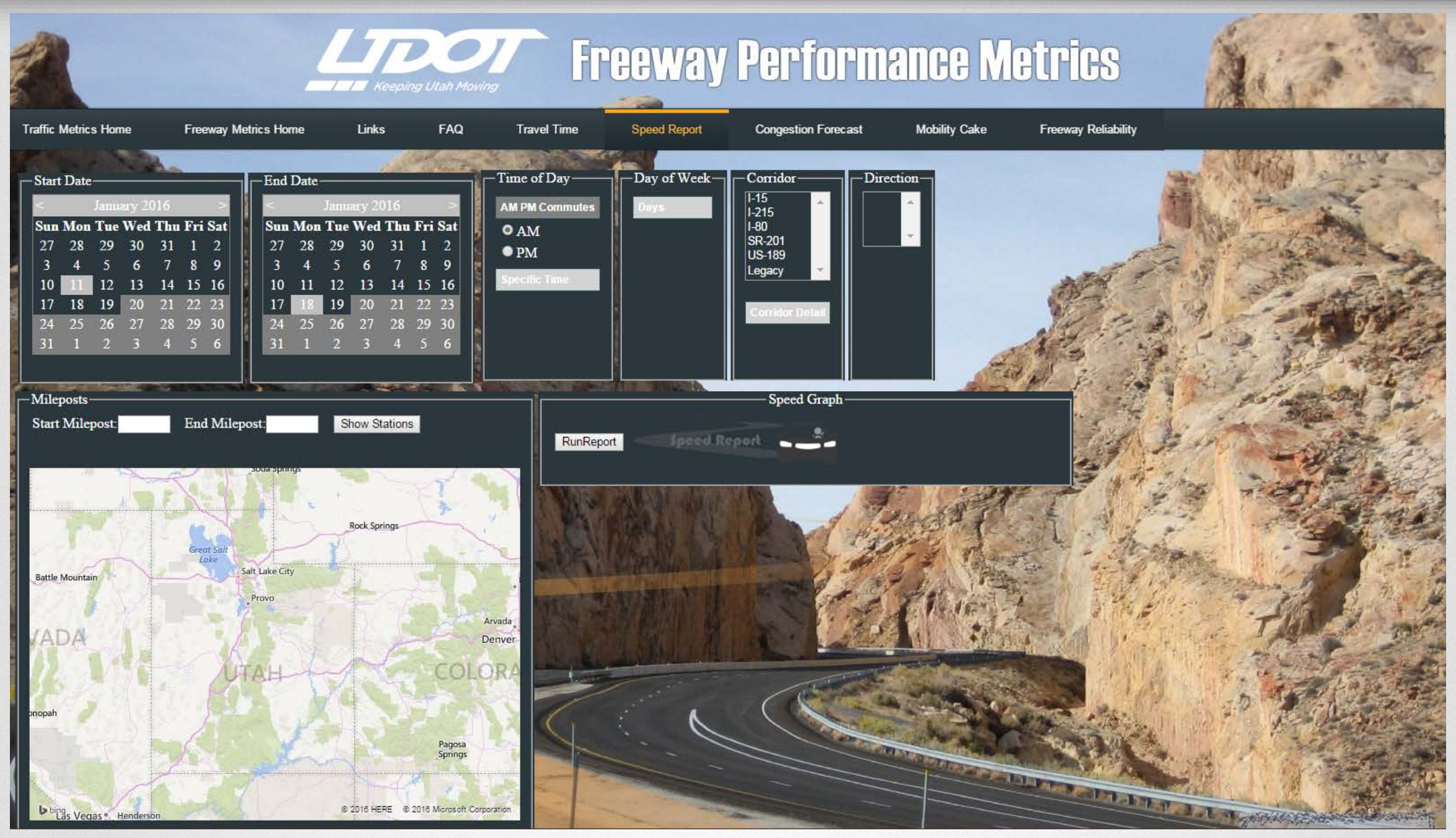

http://udottraffic.utah.gov/freewayperformancemetrics/ 


\section{Building Freeway Performance Measures}

- Brainstorm -

- What is needed

- What are we trying to measure

- What actions can we take from this metric

- Create a prototype

- Present to senior leadership

- Make adjustments

- Document process

- Allow inhouse software developers to use their creativity 


\section{Speed Report - Created with Adobe}
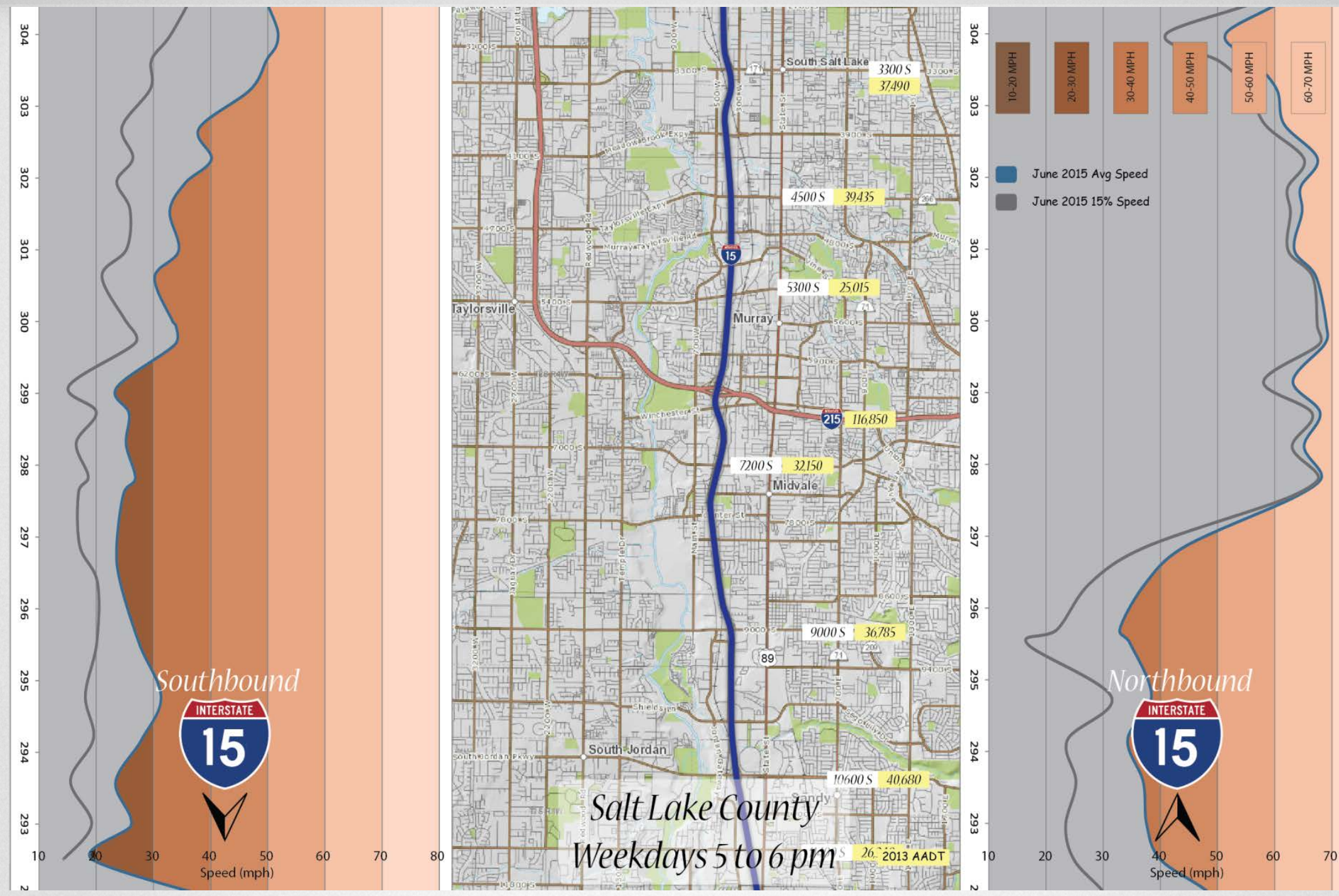


\section{Automated Speed Report}

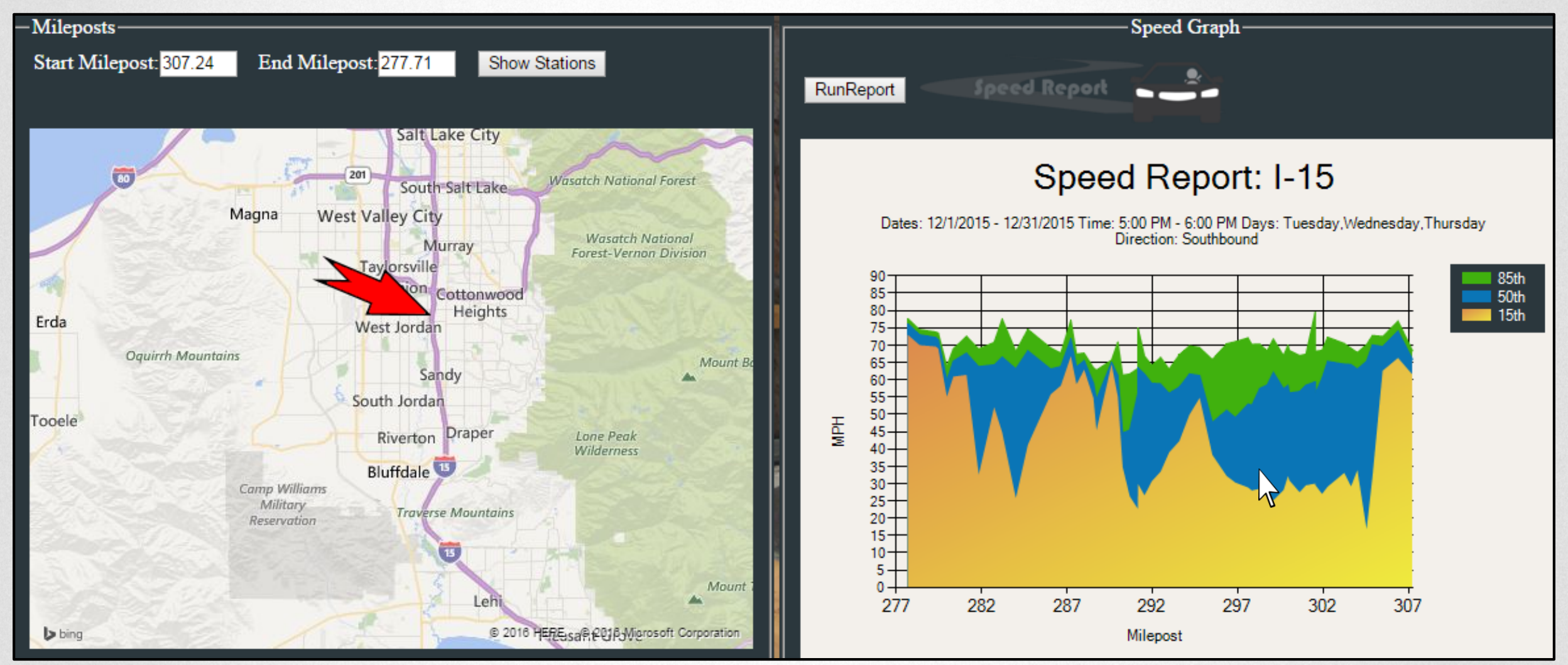




\section{Mobility Cake}

- Show the delay that can be attributed to the incidents, construction, and weather.

- Show where the delay is occurring on a corridor.

- Make assumptions that can be easily understood - don't be a black box algorithm.

- Leverage existing databases and ITS infrastructure

I-15 SB Delay: SLC to SR-92

December 2013 (All Day, Every Day)
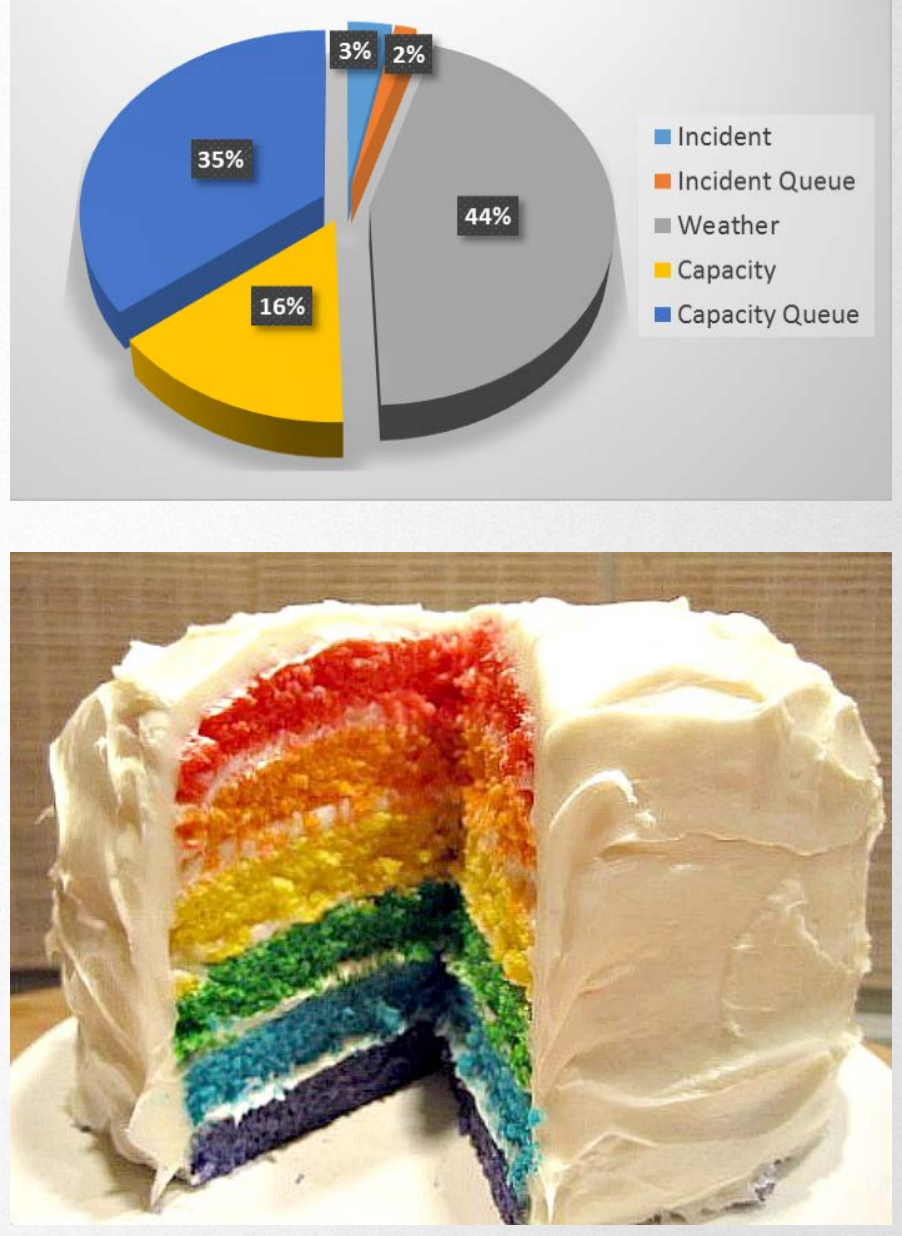


\section{Mobility Cake}
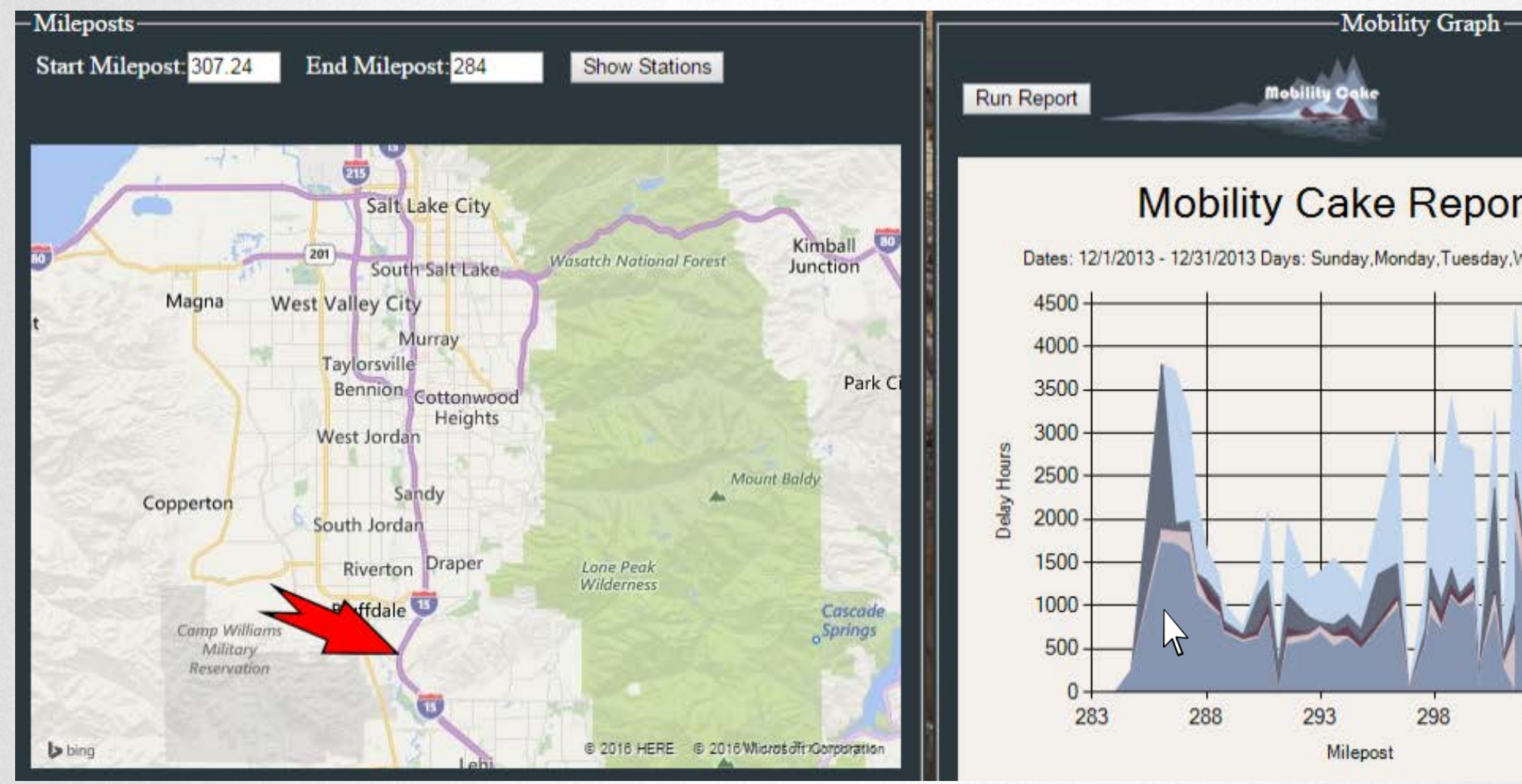

\section{Mobility Cake Report: I-15 SB}

Dates: 12/1/2013 - 12/31/2013 Days: Sunday,Monday,Tuesday,Wednesday, Thursday,Friday,Saturday

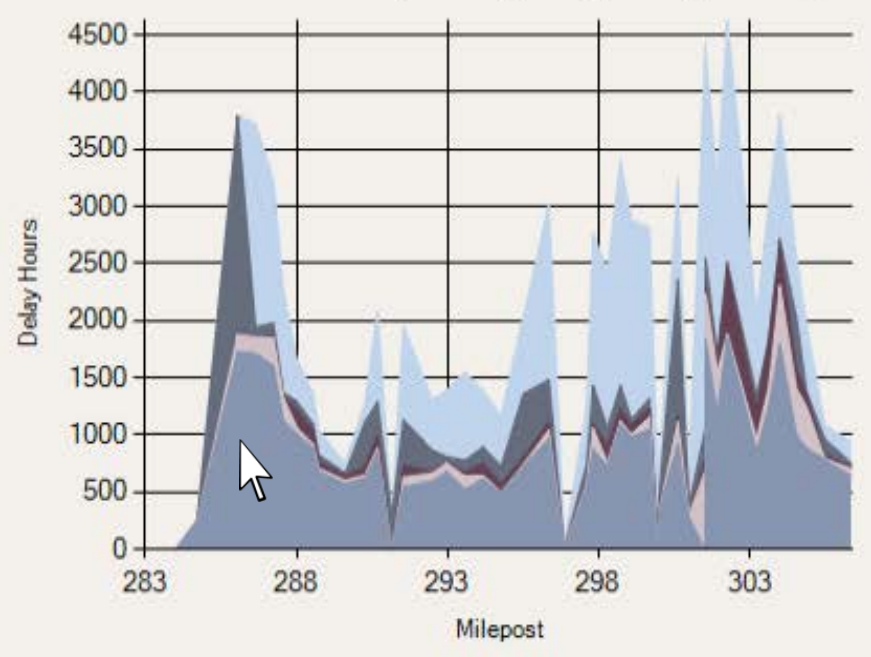

- Capacityqueue

Capacity

IncidentQueue

Incident 


\section{UDOT Automated Performance Metrics}

1. Online

- $\quad$ Speed Report

- Travel Time

2. Staging

- Mobility Cake

- Traffic/Incident Time-Lapse

- Reliability

3. Future

- Delay

- Volume-Balance

- Ramp Meter Wait Time

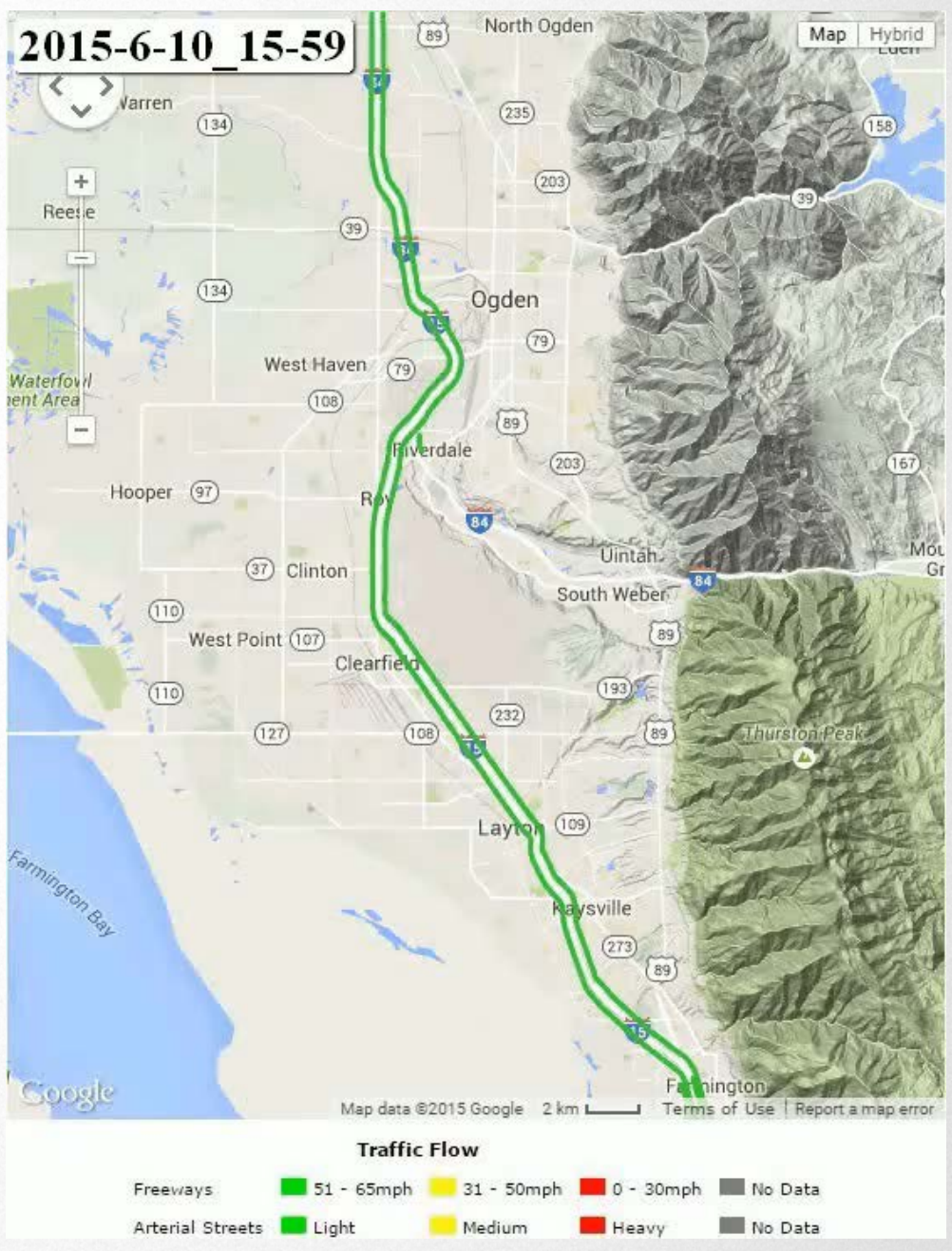


Grant Farnsworth gfarnsworth@utah.gov

udottraffic.utah.gov/performancemetrics

Questions? 